\title{
Weather in stellar atmosphere revealed by the dynamics of mercury clouds in $\alpha$ Andromedae
}

\author{
OLEG KOCHUKHOV ${ }^{1 *}$, SAUL J. ADELMAN² ${ }^{2}$, AUSTIN F. GULLIVER ${ }^{3}$ AND NIKOLAI PISKUNOV ${ }^{1}$ \\ ${ }^{1}$ Department of Astronomy and Space Physics, Uppsala University, Box 515, SE 75120 Uppsala, Sweden \\ ${ }^{2}$ Department of Physics, The Citadel, 171 Moultrie Street, 29409 Charleston, South Carolina, USA \\ ${ }^{3}$ Department of Physics and Astronomy, Brandon University, R7A 6A9 Brandon, Manitoba, Canada \\ *e-mail: Oleg.Kochukhov@astro.uu.se
}

The formation of long-lasting structures at the surfaces of stars is commonly ascribed to the action of strong magnetic fields. This paradigm is supported by observations of evolving cool spots in the Sun ${ }^{1}$ and active late-type stars ${ }^{2}$, and stationary chemical spots in the early-type magnetic stars ${ }^{3}$. However, results of our seven-year monitoring of mercury spots in non-magnetic earlytype star $\alpha$ Andromedae show that the picture of magnetically driven structure formation is fundamentally incomplete. Using an indirect stellar-surface mapping technique, we construct a series of two-dimensional images of starspots and discover a secular evolution of the mercury cloud cover in this star. This remarkable structure-formation process, observed for the first time in any star, is plausibly attributed to a non-equilibrium, dynamical evolution of the heavy-element clouds created by atomic diffusion ${ }^{4}$, and may have the same underlying physics as the weather patterns on terrestrial and giant planets.

Since the detection of sunspot magnetic fields ${ }^{5}$, it has become clear that magnetic forces are responsible for the activity and the formation of surface structure in the Sun. Strong magnetic fields, which are generated in the solar interior and brought to the surface by convective motions, inhibit the outward flow of energy, thus creating prominent dark spots with filamentary structure ${ }^{1}$. A very similar relation between magnetic fields and cool surface spots is observed in many active solar-type stars ${ }^{2}$. All these objects possess highly structured magnetic fields, which evolve on timescales from weeks to months. Stars significantly more massive than the Sun do not have an energetic surface convection zone. The lack of mixing in their atmospheres enables diffusive segregation of chemical elements to operate efficiently. The action of the competing forces of downward gravitation acceleration and the outward radiation pressure, collectively referred to as atomic diffusion ${ }^{6}$, leads to selective heavy-element enrichment of stellar atmospheres. This phenomenon of chemical peculiarity is present in most slowly rotating A- and B-type stars. The presence of strong, well-organized magnetic fields in some chemically peculiar (CP) stars alters the efficiency of the atomic transport processes, resulting in the formation of non-uniform distributions of chemical abundances over the surface and with height in their atmospheres. The fields and chemical spots in magnetic CP stars are essentially static, showing no evolutionary changes on the timescales accessible to human observers.
Despite a qualitative difference between the magnetic characteristics and related variability patterns of the low-mass solar-type stars and massive early-type objects, an undeniable and seemingly universal link between the presence of magnetic fields and the surface structure formation exists in both groups of stars. A series of recent studies has challenged this picture. A small group of late-B CP stars of mercury-manganese $(\mathrm{HgMn})$ peculiarity type has no convincing evidence of the dynamically important magnetic fields ${ }^{7}$, but, at the same time, shows clear signatures of a nonuniform surface distribution of mercury and several other heavy elements $^{8-10}$. The bright HgMn star $\alpha$ And (HR 15, HD 358) was the first non-magnetic spotted star discovered. An extensive magneticfield search ${ }^{11}$ has established at the $99 \%$ confidence level that no fields stronger than 50-100 G are present in the atmosphere of this star. This upper limit is significantly smaller than the field strength for which the magnetic and thermodynamic energy densities are equal (equipartition field, $\approx 250 \mathrm{G}$ for the lower atmospheric layers). This finding effectively rules out any magnetically driven creation of chemical spots. Thereby $\alpha$ And and other spotted HgMn stars present a formidable theoretical and observational challenge to our understanding of the formation of structure in stellar atmospheres.

The mechanism responsible for the origin of spots in nonmagnetic stars can be clarified with a comprehensive investigation, which examines the two-dimensional (2D) geometry of the surface chemical inhomogeneities and probes their possible temporal evolution. We undertook such a study for the $\mathrm{Hg}$ spots in $\alpha$ And. This star was observed in a seven-year-long campaign at the Dominion Astrophysical Observatory in Canada and the Special Astrophysical Observatory in Russia. Spectroscopic monitoring focused on the strongest $\mathrm{Hg}$ II line $(398.4 \mathrm{~nm})$ in the optical wavelength region of $\alpha$ And. This transition shows conspicuous variation in line strength and line-profile shape, caused by the rotational modulation of the geometrical aspect at which we observe a non-axisymmetric surface distribution of this chemical element.

We use the equivalent-width measurements of the $\mathrm{Hg}$ II $398.4 \mathrm{~nm}$ line to refine the rotation period of $\alpha$ And and to quantify the short- and long-term $\mathrm{Hg}$ II line-strength behaviour. The line-strength data folded with rotation phase are illustrated in Fig. 1a. The peak-to-peak amplitude of the equivalent-width 

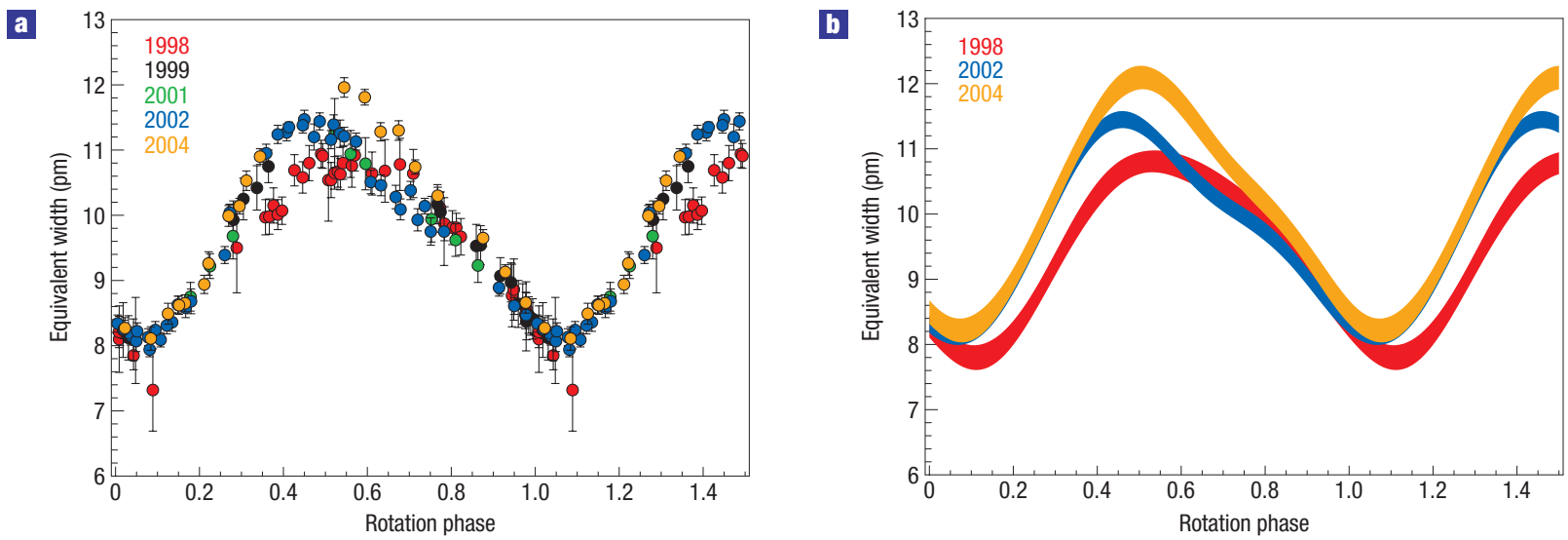

Figure $1 \mathrm{The} \mathbf{H g}$ II $398.4 \mathrm{~nm}$ line-strength variation over the rotation cycle of $\alpha$ And. a, Equivalent-width measurements with $1 \sigma$ error bars for five different epochs as a function of rotation phase. $\mathbf{b}$, A third-order Fourier fit to the line-strength variation in years 1998, 2002 and 2004. The width of the curves corresponds to a $2 \sigma$ (95\% confidence level) uncertainty of the approximating function. The significant discrepancy of the curves in the $0.3-0.7$ rotational phase interval demonstrates that the surface distribution of mercury, responsible for the $\mathrm{Hg}$ II line variability, is undergoing a slow evolution.
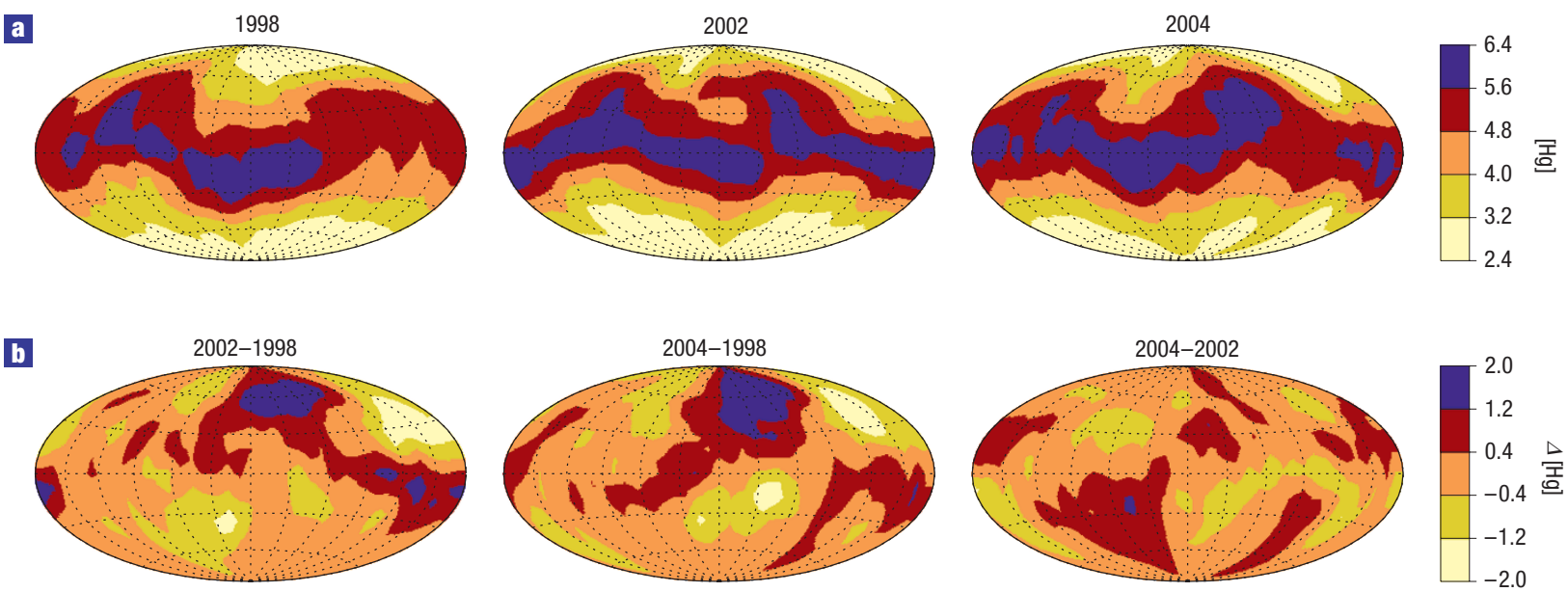

Figure 2 Distribution of the Hg clouds over the surface of $\alpha$ And in 1998, 2002 and 2004. a, The Hammer-Aitoff projection of the 2D Hg maps reconstructed with Doppler imaging. The dotted curves are lines of constant longitude and latitude, plotted every $30^{\circ}$. The longitude increases from left to right, covering the range of $0^{\circ}-360^{\circ}$. Darker regions represent higher element abundance. The $\mathrm{Hg}$ concentration is given relative to the solar value: $[\mathrm{Hg}]=\log \left(N_{\mathrm{Hg}} / N_{\mathrm{H}}\right)-\log \left(N_{\mathrm{Hg}} / N_{\mathrm{H}}\right)_{\odot}$, where $\log \left(N_{\mathrm{Hg}} / N_{\mathrm{H}}\right)_{\odot}=-10.87$ (ref. 26) and $N_{\mathrm{Hg}, \mathrm{H}}$ denote the number densities of $\mathrm{Hg}$ and $\mathrm{H}$. b. The $\mathrm{Hg}$ difference maps showing the variation of the mercury surface distribution from one epoch to another.

modulation reaches $40 \%$. The overall shape of the line-strength curve is stable over the period of seven years, but a notable change from one epoch to another is detected close to the rotation phase 0.5 , corresponding to the maximum in equivalent width. Fourier analysis (Fig. 1b) of the Hg II equivalent-width curves constructed for the three epochs with complete coverage of the rotation cycle shows that the discrepancy in the line-strength behaviour observed for different epochs is highly significant (>99\% confidence level). A complementary investigation of the $\mathrm{Hg}$ II line-profile morphology (see Supplementary Information, Fig. S1) confirms that the observed modification of the equivalent-width curve corresponds to a real change in the line-profile variability pattern. We attribute this secular variation of the $\mathrm{Hg}$ I $398.4 \mathrm{~nm}$ line-strength curve to a long-term evolution of the $\mathrm{Hg}$ spot structure in $\alpha$ And.

To obtain a complete picture of the $\mathrm{Hg}$ spot dynamics in $\alpha$ And, we applied a Doppler imaging procedure ${ }^{8}$ to the subsets of the $\mathrm{Hg}$ II $398.4 \mathrm{~nm}$ spectrograms collected in 1998, 2002 and 2004. This enabled us to construct a series of 2D maps of the stellar surface, giving insight into the evolution of the $\mathrm{Hg}$ inhomogeneities. The three independently reconstructed mercury maps are presented in Fig. 2a. The common feature of these images is a significant pole-to-equator gradient in chemical abundance. The mercury concentration at the rotation poles of $\alpha$ And exceeds the solar abundance of this element by a factor of 250 , whereas the typical overabundance in the equatorial region is $2.5 \times 10^{6}$. Several large $\left(30^{\circ}-60^{\circ}\right)$ mercury clouds at low and intermediate latitudes distort the axisymmetric pattern, leading to the observed rotational modulation of the $\mathrm{Hg}$ II line. The structure of the mercury clouds clearly changes on a timescale of $2-4$ years (Fig. 2b). In particular, a comparison of the year-1998 map with the abundance distributions derived for years 2002 and 2004 shows the emergence of a new large $\mathrm{Hg}$ spot at longitude $\approx 250^{\circ}$. At the same time, the concentration of mercury has decreased around longitude $330^{\circ}$. The amplitude of these abundance variations reaches a factor of 100 in some surface 
regions, which is comparable to the star-to-star scatter in the mean level of the $\mathrm{Hg}$ abundance in the atmospheres of HgMn stars ${ }^{12}$.

The discovery of the secular variation of the $\mathrm{Hg}$ spot geometry in $\alpha$ And opens an entirely new perspective on structure formation mechanisms in stellar atmospheres. Our analysis is the first to reveal evolving chemical inhomogeneities, apparently unrelated to magnetic fields, in any star. Radiative-diffusion calculations ${ }^{4}$ predict that $\mathrm{Hg}$ accumulates in a thin cloud in the upper atmosphere, where the gravitational force and radiative pressure nearly cancel out. Levitating $\mathrm{Hg}$ atoms become susceptible to minute external perturbations. A $1.3 \%$ decrease in the effective gravity due to the centrifugal force at the equator of $\alpha$ And corresponds to a roughly 300-times-higher $\mathrm{Hg}$ abundance. A small upward displacement of the mercury cloud converts $\mathrm{Hg}$ II atoms to the unobservable $\mathrm{Hg}$ III state, thus substantially reducing the strength of the $\mathrm{Hg}$ II $398.4 \mathrm{~nm}$ line. In this scenario, the observed time-dependent non-axisymmetric component of the chemical images is attributed to hydrodynamical instabilities that develop in the Hg-rich layer. The $\mathrm{Hg}$ cloud cover could be disrupted by the thermohaline instability induced by inverse molecular-weight gradients $^{13}$ or by the dynamic tidal perturbation ${ }^{14}$ due to the secondary star moving in a highly eccentric orbit. Alternatively, a time-dependent radiative-diffusion scenario ${ }^{15}$, involving several interacting $\mathrm{Hg}$ layers at different heights, could contribute to the variation of the chemical structures in $\alpha$ And. Independently of the theory scrutinizing details of the atmospheric diffusion instabilities, we can draw a parallel between evolving chemical-cloud 'weather' in stars and the weather patterns on the Earth and the giant gas planets. Our investigation demonstrates that, similar to planetary atmospheres, the outermost layers of stars are sites of dynamical processes of structure formation and self-organization.

The observation of the $\mathrm{Hg}$ cloud dynamics in $\alpha$ And offers a possible solution to the long-standing problem of the chemical diversity of $\mathrm{HgMn}$ and related stars. If a competition between radiative levitation and gravity, with the possible influence of weak mass $\operatorname{loss}^{16}$, is the primary mechanism responsible for the accumulation of heavy elements in the atmospheres of HgMn and similar CP stars, we would expect to find very similar abundances in stars of the same mass and evolutionary stage. Contrary to this prediction of the simple diffusion theory, a large discrepancy in the heavy ${ }^{12}$ and iron-peak ${ }^{17}$ element abundances is often reported for stars with similar fundamental parameters. We propose that the heavy-element atmospheric enrichment created by atomic diffusion is not stationary but evolves under the influence of the hydrodynamical instability of the same kind as observed in $\alpha$ And. As a result of this time-variable process, atmospheric abundances change quickly compared to the stellar evolutionary timescale. Thus, the observed concentrations of the elements most sensitive to the diffusion effect include a significant stochastic or, perhaps, a cyclic time-dependent component and are only loosely related to the stellar parameters. The mere presence and the magnitude of the characteristic chemical peculiarities may represent a transient phase in the long-term evolution of the chemical composition of the stellar surface layers.

\section{METHODS}

\section{SPECTROSCOPIC OBSERVATIONS}

We have collected 127 observations of $\alpha$ And in the $\mathrm{Hg}$ II $398.4 \mathrm{~nm}$ region during five different epochs, spanning from 10 days to 4 months, over the period of seven years. Observations were obtained with the high-resolution spectrographs attached to the $1.2 \mathrm{~m}$ telescope of the Dominion Astrophysical Observatory (Canada) and the $6 \mathrm{~m}$ telescope of the Special Astrophysical Observatory (Russia). The spectra have resolving powers of

$\lambda / \Delta \lambda=36,500-44,000$ and signal-to-noise ratios between 500:1 and 1,500:1.
Reduction of the spectrograms used our extraction programs ${ }^{18,19}$ to optimize the signal-to-noise ratio of the $\alpha$ And observations.

\section{TIME-SERIES ANALYSIS}

Confirming results of the previous studies ${ }^{8}$, we find that the $\mathrm{Hg}$ II $398.4 \mathrm{~nm}$ line shows remarkable variability in the average intensity and profile shape on a timescale of $\approx 2.38 \mathrm{~d}$. Lines of other chemical elements show no evidence of the modulation caused by surface spots. The stellar rotation period of $2.38195 \pm 0.00003 \mathrm{~d}$ is inferred from the changes in $\mathrm{Hg}$ II equivalent width. Two independent time-series analysis methods, a third-order least-squares Fourier fitting and phase dispersion minimization ${ }^{20}$, yield consistent values of the rotation period.

\section{STELLAR PARAMETERS}

$\alpha$ And is a spectroscopic binary star with a spotted HgMn B8p primary and an A3m secondary ${ }^{21,22}$. The orbital period is $96.70 \mathrm{~d}$. The atmospheric parameters ${ }^{22}$ essential for modelling the spectra of the system are effective temperature $T_{\text {eff }}=13,800 \mathrm{~K}$, surface gravity $\log g=3.75$ for the primary and $T_{\text {eff }}=8,500 \mathrm{~K}, \log g=4.0$ for the secondary. The less massive star is $2 \mathrm{mag}$ fainter than the primary and has a much higher projected rotational velocity. The contribution from its line absorption is negligible at the location of the $\mathrm{Hg}$ II $398.4 \mathrm{~nm}$ feature. However, the secondary's contribution to the continuum radiation weakens the lines of the primary star. We compensate for this effect by correcting the observed spectra: $S_{\text {cor }}=S(1+1 / R)-1 / R$, where the luminosity ratio $R \equiv L_{\text {prim }} / L_{\text {sec }}=8.72$ at $398.4 \mathrm{~nm}$.

\section{DOPPLER IMAGING}

We reconstruct the distribution of the $\mathrm{Hg}$ abundance over the surface of $\alpha$ And with the help of Doppler imaging ${ }^{23}$. This technique takes advantage of the partial resolution of the stellar surface provided by the rotational Doppler effect, inverting a line-profile time series into a 2D map of the stellar surface. A regularization procedure ${ }^{24}$ is used to ensure that a unique, simplest possible map is derived. The abundance Doppler imaging code INVERS12 (ref. 3 ) is applied to interpret the observations of $\alpha$ And. The stellar line profiles are computed on a $(30 \times 60)$-element surface grid, using the ATLAS9 $($ ref. 25$)$ model atmosphere. The projected rotational velocity, $v \sin i=53.0 \pm 0.5 \mathrm{~km} \mathrm{~s}^{-1}$, is determined from the line-profile fit, and the inclination angle $i=75^{\circ}$ is adopted following refs 8,21 . The line list used for spectrum synthesis calculations includes 15 isotope and hyperfine components ${ }^{12}$ of the $\mathrm{Hg}$ II $398.4 \mathrm{~nm}$ transition and the Y II $398.26 \mathrm{~nm}$ line, which blends with the blue wing of the $\mathrm{Hg}$ II feature. The hydrogen bound-bound opacity due to the $\mathrm{H} \varepsilon$ line at $397 \mathrm{~nm}$ is also accounted for. In our surface-mapping procedure the $\mathrm{Hg}$ isotope mixture is adjusted simultaneously with the total abundance of this element. We find that the isotope composition of $\mathrm{Hg}$ in $\alpha$ And does not deviate significantly from the solar mixture and varies little over the surface of the star. A comparison of the observed and computed $\mathrm{Hg}$ II line profiles (see Supplementary Information, Fig. S2) indicates that our models of the $\mathrm{Hg}$ surface spots yield a good fit to observations for all three epochs considered. Additional numerical tests summarized in Supplementary Information, Fig. S3 confirm that the variation of the $\mathrm{Hg}$ spot structure from one epoch to another is highly significant and cannot be attributed to the difference in data quality and rotational phase coverage.

\section{Received 3 April 2007; accepted 30 May 2007; published 24 June 2007.}

\section{References}

1. Scharmer, G. B., Gudiksen, B. V., Kiselman, D., Löfdahl, M. G. \& Rouppe van der Voort, L. H. M. Dark cores in sunspot penumbral filaments. Nature 420, 151-153 (2002).

2. Donati, J.-F. et al. Dynamo processes and activity cycles of the active stars AB Doradus, LQ Hydrae and HR 1099. Monthly Notices of RAS 345, 1145-1186 (2003).

3. Kochukhov, O., Drake, N. A., Piskunov, N. \& de la Reza, R. Multi-element abundance Doppler imaging of the rapidly oscillating Ap star HR 3831. Astron. Astrophys. 424, 935-950 (2004).

4. Michaud, G., Reeves, H. \& Charland, Y. Diffusion and isotope anomalies of Hg in Ap stars. Astron. Astrophys. 37, 313-324 (1974).

5. Hale, G. E. On the probable existence of a magnetic field in sun-spots. Astrophys. J. 28 315-343 (1908).

6. Michaud, G. Diffusion processes in peculiar A stars. Astrophys. J. 160, 641-658 (1970).

7. Shorlin, S. L. S. et al. A highly sensitive search for magnetic fields in B, A and F stars. Astron. Astrophys. 392, 637-652 (2002).

8. Adelman, S. J., Gulliver, A. F., Kochukhov, O. P. \& Ryabchikova, T. A. The variability of the Hg II $\lambda 3984$ line of the mercury-manganese star $\alpha$ Andromedae. Astrophys. J. 575, 449-460 (2002).

9. Kochukhov, O., Piskunov, N., Sachkov, M. \& Kudryavtsev, D. Inhomogeneous distribution of mercury on the surfaces of rapidly rotating HgMn stars. Astron. Astrophys. 439, 1093-1098 (2005). 
10. Hubrig, S. et al. Inhomogeneous surface distribution of chemical elements in the eclipsing binary AR Aur: A new challenge for our understanding of HgMn stars. Monthly Notices of RAS 371, 1953-1958 (2006).

11. Wade, G. A. et al. A search for magnetic fields in the variable $\mathrm{HgMn}$ star $\alpha$ Andromedae. Astron. Astrophys. 451, 293-302 (2006).

12. Woolf, V. M. \& Lambert, D. L. Mercury elemental and isotopic abundances in mercury-manganese stars. Astrophys. J. 521, 414-431 (1999).

13. Vauclair, S. Metallic fingers and metallicity excess in exoplanets' host stars: The accretion hypothesis revisited. Astrophys. J. 605, 874-879 (2004).

14. Willems, B. \& Aerts, C. Tidally induced radial-velocity variations in close binaries. Astron. Astrophys. 384, 441-451 (2002).

15. Alecian, G. Time-dependent diffusion in A-stars. Contributions of the Astronomical Observatory Skalnate Pleso 27, 290-295 (1998)

16. Proffitt, C. R. et al. Mercury in the HgMn Stars $\chi$ Lupi and HR 7775. Astrophys. J. 512, 942-960 (1999).

17. Smith, K. C. \& Dworetsky, M. M. Elemental abundances in normal late B-stars and HgMn-stars from co-added IUE spectra. I. Iron peak elements. Astron. Astrophys. 274, 335-355 (1993).

18. Gulliver, A. F. \& Hill, G. in ASP Conf. Ser. 281: Astronomical Data Analysis Software and Systems X (eds Bohlender, D. A., Durand, D. \& Handley, T. H.) 351-354 (Astronomical Society of the Pacific, San Francisco, 2002)

19. Piskunov, N. E. \& Valenti, J. A. New algorithms for reducing cross-dispersed echelle spectra. Astron. Astrophys. 385, 1095-1106 (2002)

20. Stellingwerf, R. F. Period determination using phase dispersion minimization. Astrophys. J. 224, 953-960 (1978)

21. Pan, X. et al. Determination of the visual orbit of the spectroscopic binary $\alpha$ Andromedae with submilliarcsecond precision. Astrophys. J. 384, 624-633 (1992).

22. Ryabchikova, T. A., Malanushenko, V. P. \& Adelman, S. J. Orbital elements and abundance analyses of the double-lined spectroscopic binary $\alpha$ Andromedae. Astron. Astrophys. 351, 963-972 (1999).
23. Rice, J. B. Doppler imaging of stellar surfaces-techniques and issues. Astronomische Nachrichten 323, 220-235 (2002).

24. Piskunov, N. E. \& Rice, J. B. Techniques for surface imaging of stars. Publ. Astron. Soc. Pac. 105, 1415-1421 (1993).

25. Kurucz, R. L. ATLAS9 Stellar Atmosphere Programs and 2 km/s grid. Kurucz CD-ROM No. 13. (Smithsonian Astrophysical Observatory, Cambridge, 1993).

26. Asplund, M., Grevesse, N. \& Sauval, A. J. in ASP Conf. Ser. 336: Cosmic Abundances as Records of Stellar Evolution and Nucleosynthesis (eds Barnes, T. G. III \& Bash, F. N.) 25-38 (Astronomical Society of the Pacific, San Francisco, 2005

\section{Acknowledgements}

O.K. and N.P. acknowledge financial support from the Swedish Research Council and the Royal Academy of Sciences. M. Sachkov and D. Kudryavtsev are thanked for their assistance with observations of $\alpha$ And at the $6 \mathrm{~m} \mathrm{SAO}$ telescope. S.J.A. and A.F.G. are Guest Investigators at the Dominion Astrophysical Observatory operated by the Herzberg Institute of Astrophysics, National Research Council of Canada. They thank J.E. Hesser, Director of the Dominion Astrophysical Observatory, for the observing time. S.J.A.'s contribution to this paper was supported in part by grants from The Citadel Foundation. Financial support was provided to A.F.G. by the Natural Sciences and Engineering Research Council of Canada.

Correspondence and requests for materials should be addressed to O.K.

Supplementary Information accompanies this paper on www.nature.com/naturephysics.

\section{Competing financial interests}

The authors declare no competing financial interests.

Reprints and permission information is available online at http://npg.nature.com/reprintsandpermissions/ 